\title{
Modelagem agrometeorológica do rendimento de arroz irrigado no Rio Grande do Sul
}

\author{
Eliana Veleda Klering ${ }^{(1)}$, Denise Cybis Fontana( ${ }^{(2)}$, Moacir Antonio Berlato ${ }^{(2)}$ e Alberto Cargnelutti Filho ${ }^{(3)}$
}

\begin{abstract}
(1)Universidade Federal do Rio Grande do Sul (UFRGS), Centro Estadual de Pesquisas em Sensoriamento Remoto e Meteorologia, Avenida Bento Gonçalves, no 9500, Caixa Postal 15044, CEP 91501-970 Porto Alegre, RS. E-mail: elianaklering@yahoo.com.br (2)UFRGS, Departamento de Plantas Forrageiras e Agrometeorologia, Avenida Bento Gonçalves, no 7712, Caixa Postal 15100, CEP 91501-970 Porto Alegre, RS. E-mail: dfontana@ufrgs.br, moacir.berlato@ufrgs.br(3)UFRGS, Departamento de Estatística, CEP 91509-900 Porto Alegre, RS. E-mail: cargnelutti@ufrgs.br
\end{abstract}

\begin{abstract}
Resumo - O objetivo deste trabalho foi elaborar e testar modelos para a estimativa de rendimento de arroz irrigado, no Estado do Rio Grande do Sul. O estudo foi realizado com dados meteorológicos de temperatura mínima do ar, radiação solar global e dados de estatísticas agrícolas de rendimento de arroz irrigado, das seis regiões orizícolas do Rio Grande do Sul, referentes às safras 1982/1983 até 2005/2006. Foram feitas análises de tendência tecnológica dos rendimentos, e foram estabelecidos os indicadores agrometeorológicos para o ajuste de modelos de estimativa de rendimento de arroz irrigado, para o Rio Grande do Sul. Existe tendência tecnológica de aumento nos rendimentos de arroz irrigado no Estado. As variáveis meteorológicas avaliadas - dias com temperatura mínima do ar inferior a $15^{\circ} \mathrm{C}$ e radiação solar global - podem ser usadas como indicadores do rendimento de arroz irrigado. Os modelos agrometeorológicos elaborados para as seis regiões orizícolas e para o Estado do Rio Grande do Sul apresentam características de precisão, fácil implementação e baixo custo e podem, portanto, ser introduzidos ao programa nacional de previsão de safras.
\end{abstract}

Termos para indexação: Oryza sativa, previsão de safras, radiação solar, temperatura mínima.

\section{Agrometeorological modelling of irrigated rice yield in Rio Grande do Sul, Brazil}

\begin{abstract}
The objective of this work was to elaborate and test models to estimate the irrigated rice yield, in Rio Grande do Sul State, Brazil. The study was carried out using meteorological data of minimum air temperature, global solar radiation and data of agricultural statistics about the irrigated rice yield, involving six rice production regions of Rio Grande do Sul, relative to crop years from 1982/1983 to 2005/2006. Analyses of yield technological tendencies were performed, and agrometeorological indicators for model adjustments of irrigated rice yields were established. There is a technological tendency of increasing the irrigated rice yield in the State. The analyzed meteorological variables - global solar radiation and days with minimum air temperature below or equal to $15^{\circ} \mathrm{C}$ - can be used as indicators of the irrigated rice yield. The adjusted agrometeorological models, elaborated for the six rice production regions and for the Rio Grande do Sul State, show characteristics of accuracy, easy implementation and low cost, which make them able to be introduced in the national program of crop forecast.
\end{abstract}

Index terms: Oryza sativa, crop forecast, solar radiation, minimum temperature.

\section{Introdução}

O arroz é um dos cereais mais produzidos e consumidos no mundo e cultivado em todos os continentes (Azambuja et al., 2004). Atualmente, o Rio Grande do Sul é considerado estabilizador da safra nacional, pois produz $48 \%$ da produção brasileira. Isto representa 3,1\% do PIB e gera R 175 milhões em imposto de circulação de mercadorias e serviços (ICMS) e 250 mil empregos no Estado (IBGE, 2004).
A previsão das safras de arroz no Estado, assim como de diversas culturas em todo o Brasil, é realizada com uso de informações municipais, obtidas por meio de um sistema de levantamento, baseado em opiniões de agentes técnicos e econômicos relacionados ao setor (IBGE, 2004). Contudo, em função do caráter subjetivo dos levantamentos, essas informações não permitem uma análise quantitativa dos erros envolvidos, além de serem passíveis de manipulação. É, portanto, de grande importância o desenvolvimento de métodos objetivos que 
possam ser incorporados, em complementação aos programas oficiais.

Uma das alternativas para a resolução do problema da subjetividade do método oficial é o uso de modelos de estimativa de rendimento, os quais, em sua maioria, expressam a influência de elementos meteorológicos no rendimento das culturas agrícolas, e são denominados modelos agrometeorológicos. Alguns exemplos de trabalhos realizados no Estado são os de Matzenauer et al. (1995), para o milho, e Carmona et al. (2002) para o arroz.

Oldeman et al. (1986) demonstraram que o arroz cultivado em áreas inundadas, onde a disponibilidade de água não restringe o crescimento e o desenvolvimento da cultura, e onde os estresses biológicos e as condições adversas do solo são mínimos, o rendimento potencial está relacionado, principalmente, à temperatura do ar e à radiação solar global.

Apesar de não poder ser considerada isoladamente, a temperatura do ar é um dos elementos meteorológicos que exerce maior influência sobre o crescimento, desenvolvimento e rendimento do arroz. Em geral, a cultura exige temperaturas relativamente elevadas da germinação à maturação, uniformemente crescentes até a floração, e decrescentes - porém sem diminuições bruscas - após a floração (Yoshida, 1981, citado por Steinmetz, 2004). As plantas de arroz são mais sensíveis aos efeitos das baixas temperaturas nas fases de préfloração e floração, em que a esterilidade das espiguetas pode causar decréscimos acentuados de rendimento (Mota, 1994). A temperatura crítica de $15^{\circ} \mathrm{C}$, para toda a fase reprodutiva, tem sido usada como referência em estudos climatológicos para o arroz irrigado do Estado do Rio Grande do Sul (Steinmetz et al., 2001).

A exigência de radiação solar pela cultura do arroz varia de uma fase fenológica para a outra. Durante a fase vegetativa, a radiação solar tem relativamente pouca influência sobre o rendimento e os seus componentes. Entretanto, os índices de radiação solar, durante as fases reprodutiva e de maturação, influenciam fortemente os rendimentos de arroz irrigado (Yoshida \& Parao, 1976).

Já que a temperatura do ar e a radiação solar global estão associadas ao rendimento da cultura do arroz irrigado e estão disponíveis em estações meteorológicas, essas duas variáveis podem compor um modelo consistente para estimar o rendimento desta cultura.

O objetivo deste trabalho foi elaborar e testar modelos para a estimativa de rendimento de arroz irrigado, para o Estado do Rio Grande do Sul.

\section{Material e Métodos}

A área de estudo deste trabalho é a região orizícola do Estado do Rio Grande do Sul que é dividida em: Fronteira Oeste, Campanha, Depressão Central, Planície Costeira Interna à Lagoa dos Patos, Planície Costeira Externa à Lagoa dos Patos e Zona Sul (Figura 1).

Nas análises, foram utilizados dados oficiais de rendimento da cultura do arroz irrigado de 24 safras agrícolas (1982/1983 a 2005/2006), obtidos do sítio de Levantamento Sistemático da Produção Agrícola (LSPA) do Instituto Brasileiro de Geografia e Estatística (IBGE, 2004).

Utilizaram-se, também, dados meteorológicos diários e mensais de temperatura mínima do ar e radiação solar global das 17 estações meteorológicas da região de estudo (Figura 1).

$\mathrm{O}$ instrumento usado para medição da radiação solar foi o actinógrafo, geralmente disponível nas estações agrometeorológicas da Fundação Estadual de Pesquisa Agropecuária/Secretaria Estadual da Ciência e Tecnologia do Estado do Rio Grande do Sul (Fepagro/SCT-RS). $\mathrm{Na}$ inexistência desses dados - o que ocorreu na maior parte das estações do 8o Distrito de Meteorologia, do Instituto Nacional de Meteorologia (8o Disme/Inmet) -, usou-se o heliógrafo, que mede a insolação (horas de sol por dia). Nesses casos, para estimar a radiação solar global foi utilizada a equação de Ångstrom dada por:

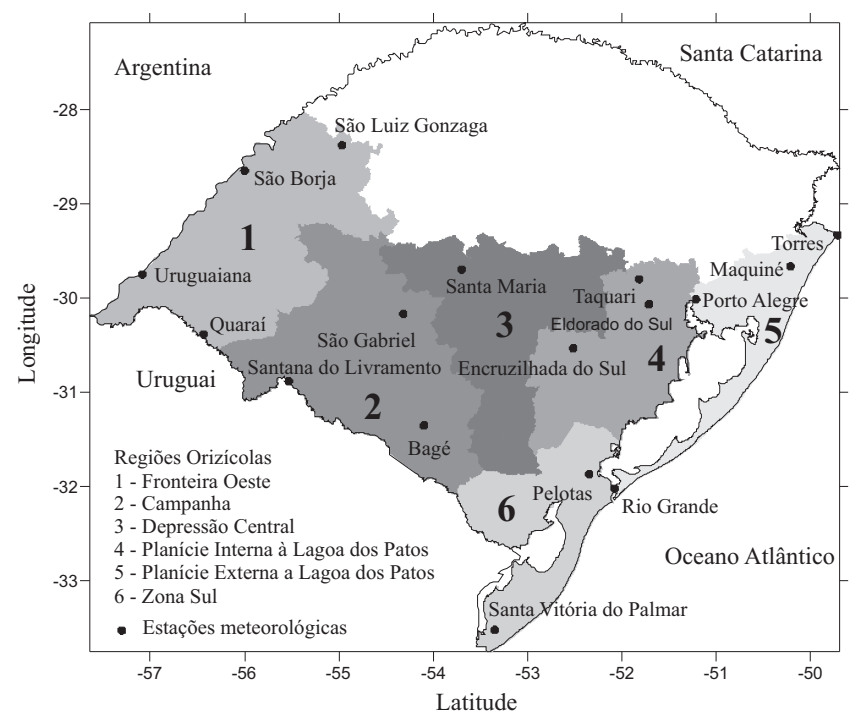

Figura 1. Regiões orizícolas do Estado do Rio Grande do Sul e estações meteorológicas utilizadas no estudo. 


$$
\frac{\mathrm{Rg}}{\mathrm{Ra}}=\beta_{0}+\beta_{1}\left(\frac{\mathrm{n}}{\mathrm{N}}\right)
$$

em que: Rg é o fluxo de radiação solar global (cal cm $\mathrm{cm}^{-2}$ por dia); Ra é a radiação solar incidente numa superfície horizontal no topo da atmosfera, paralela à superfície do solo (cal $\mathrm{cm}^{-2}$ por dia); n é o número de horas de sol ocorrido; $\mathrm{N}$ é o número de horas de sol astronomicamente possível de ocorrer; $\beta_{0}$ e $\beta_{1}$ são os coeficientes empíricos, obtidos por análise de regressão, para determinada localidade ou região (Fontana \& Oliveira, 1996).

A partir do conjunto de dados de rendimento, verificouse a existência de tendência temporal, ou seja, existência ou não de tendência de aumento dos rendimentos da cultura do arroz, em razão da incorporação de novas tecnologias (cultivares, práticas de manejo etc.) ao longo do período de estudo. A equação ajustada foi uma regressão linear simples, dada por:

$\mathrm{Y}_{\mathrm{i}}=\beta_{0}+\beta_{1} \mathrm{x}_{\mathrm{i}}$

em que: $\mathrm{Y}_{\mathrm{i}}$ é o rendimento de grãos de arroz irrigado; $\beta_{0}$ é o coeficiente linear (intercepto); $\beta_{1}$ é o coeficiente de regressão da variável independente; $\mathrm{x}_{\mathrm{i}}$ é o período observado.

Para se constatar a existência de tendência temporal, foi feito o teste t de Student, a $1 \%$ de probabilidade, para o coeficiente de regressão $\beta_{1}$, para testar as hipóteses: $\beta_{1}=0$, não existe tendência; e $\beta_{1}>0$, existe tendência de crescimento.

As séries históricas de rendimento das seis regiões orizícolas do Estado foram corrigidas por meio da retirada da tendência tecnológica, inversão da Equação 2 e aplicação dos parâmetros gerados no ajuste da equação, para cada região orizícola:

$\mathrm{Y}_{\mathrm{ci}}=\mathrm{Y}_{\mathrm{i}}-\beta_{1} \mathrm{x}_{\mathrm{i}}$

em que: $\mathrm{Y}_{\mathrm{ci}}$ é o rendimento corrigido do ano $\mathrm{i} ; \mathrm{Y}_{\mathrm{i}}$ é o rendimento original do ano $\mathrm{i} ; \beta_{1}$ é o coeficiente de regressão linear; $x_{i}$ é o ano $\mathrm{i}$.

Foram analisadas as correlações entre número de dias com temperatura mínima, menor ou igual a $15^{\circ} \mathrm{C}$, e radiação solar global mensal e o rendimento corrigido, nas seis regiões orizícolas e no Estado como um todo, para as safras de 1982/1983 até 2005/2006. Verificou-se a significância dos coeficientes de correlação, por meio do teste t de Student a 1, 5 e $10 \%$ de probabilidade.
Ajustaram-se modelos agrometeorológicos de estimativa de rendimento de arroz irrigado, para as regiões orizícolas individualmente e para o conjunto delas (metade sul do Estado do Rio Grande do Sul); este último foi considerado o modelo para o Estado. Esses modelos foram ajustados por meio de regressão linear múltipla, com $\mathrm{k}$ variáveis independentes, dada por:

$\mathrm{Y}_{\mathrm{i}}=\beta_{0}+\sum_{\mathrm{i}=1}^{\mathrm{k}} \beta_{\mathrm{i}} \mathrm{X}_{\mathrm{ij}}+\varepsilon_{\mathrm{j}}$

em que: $\mathrm{Y}_{\mathrm{i}}$ é o valor corrigido do rendimento de grãos; $\beta_{0}$ é o coeficiente linear; $\beta_{i}$ é o coeficiente de regressão das variáveis independentes; $X_{i}$ e $X_{i j}$ são as variáveis independentes $X_{i}$ na observação $j$; $\varepsilon_{j}$ é o erro associado à variável Y na observação j.

Nesta equação, se pressupõe que a variável Y seja função linear das variáveis independentes, os valores das variáveis independentes são fixos, os erros têm médias zero, são homocedásticos, independentes e de distribuição normal (Neter et al., 1997; Hoffmann \& Vieira, 1998). Os parâmetros do modelo das equações de regressão linear múltipla foram estimados pelo método passo-a-passo "stepwise", com 10\% de significância para entrada e saída de variáveis, que tem como variável dependente o rendimento corrigido (após a retirada da tendência tecnológica) de arroz irrigado, e como variáveis independentes: número de dias com temperatura igual ou inferior a $15^{\circ} \mathrm{C}$, nos períodos mensais (dezembro, janeiro, fevereiro e março), bimestrais (dezembro a janeiro, janeiro a fevereiro e fevereiro a março) e trimestrais (dezembro a fevereiro e janeiro a março); radiação solar global nos períodos mensais (outubro, novembro, dezembro, janeiro, fevereiro e março), bimestrais (outubro e novembro, novembro e dezembro, dezembro e janeiro, janeiro e fevereiro, fevereiro e março), trimestrais (outubro a dezembro, novembro a janeiro, dezembro a fevereiro, janeiro a março), e quadrimestrais (outubro a janeiro, novembro a fevereiro e dezembro a março).

O período utilizado para o ajuste foi de 1982/1983 a 2005/2006, com exceção de quatro safras, escolhidas aleatoriamente, (1992/1993 até 1995/1996) as quais foram utilizadas para a validação dos modelos.

As significâncias dos coeficientes de correlação de Pearson (r), entre os rendimentos estimados pelos diferentes modelos e os rendimentos oficiais, foram verificadas por meio do teste $\mathrm{t}$ de Student, a $5 \%$ de probabilidade. 


\section{Resultados e Discussão}

Todas as regiões orizícolas e também o Estado do Rio Grande do Sul, no período de 1982 a 2006, apresentaram tendências tecnológicas embutidas no rendimento (coeficiente de regressão linear significativo a $1 \%)$. As regiões da Planície Interna $\left(\beta_{1}=0,0591 \mathrm{t} \mathrm{ha}^{-1}\right)$, Planície Externa à Lagoa dos Patos $\left(\beta_{1}=0,0393 \mathrm{t} \mathrm{ha}^{-1}\right) \mathrm{e}$ Zona Sul $\left(\beta_{1}=0,0422 \mathrm{t} \mathrm{ha}^{-1}\right)$ foram as que apresentaram os menores incrementos; as regiões da Depressão Central $\left(\beta_{1}=0,0643 \mathrm{tha}^{-1}\right)$ e Campanha $\left(\beta_{1}=0,0825 \mathrm{t} \mathrm{ha}^{-1}\right)$ apresentaram níveis intermediários; e a região que apresentou o maior nível tecnológico foi a Fronteira Oeste $\left(\beta_{1}=0,1081 \mathrm{t} \mathrm{ha}^{-1}\right)$ (Figura 2). Estes resultados diferem dos encontrados por Carmona et al. (2002), que verificou tendência semelhante para as seis regiões orizícolas do Estado. As análises, entretanto, foram feitas para um período de estudo maior (1944-2000).

A tendência temporal, para o rendimento de arroz irrigado no Estado, mostrou aumento de $0,0659 \mathrm{t} \mathrm{ha}^{-1} \mathrm{a}$ cada ano, nos rendimentos do período avaliado, fato que pode ser explicado pela adoção de novas tecnologias no sistema de produção da cultura, como o uso de novas cultivares e insumos, assim como de manejos mais eficientes, entre outros. O coeficiente de determinação $\left(\mathrm{R}^{2}\right)$ da equação ajustada para o Estado, mostra que os avanços tecnológicos responderam por cerca de 55\% da variabilidade dos rendimentos de arroz irrigado. Podese assumir, portanto, que após a retirada da tendência tecnológica, as variações interanuais no rendimento de arroz estão associadas a variações nas condições ambientais, as quais deverão explicar o restante da variabilidade dos rendimentos desta cultura. Para a série analisada, as condições ambientais, em razão principalmente das condições meteorológicas e de manejo da cultura, determinaram uma flutuação de $-1,2$ a $+0,66 \mathrm{t} \mathrm{ha}^{-1}$. A separação dos efeitos ocasionados por uma ou outra causa de variação não são possíveis de identificação na análise feita neste trabalho.

A primeira etapa na busca de um modelo, para a estimativa do rendimento do arroz irrigado no Rio Grande do Sul, foi a identificação de suas variáveis de entrada; neste trabalho, foram avaliadas a temperatura mínima do ar e a radiação solar global.

Os coeficientes de correlação entre o número de dias com temperatura mínima igual ou inferior a $15^{\circ} \mathrm{C}$ e o rendimento (Tabela 1), com exceção das regiões da Depressão Central (Região 3) e Zona Sul (Região 6), foram negativos em todas as regiões orizícolas e também para o Estado do Rio Grande do Sul. Isto demonstra que maior ocorrência de dias com temperaturas mínimas baixas é um fator que pode penalizar o rendimento, dado também apontado por Carmona et al. (2002). O mês mais crítico foi março, em que foram observados os maiores valores de correlação na maior parte das regiões orizícolas e, também, para o Estado como um todo. Em outras combinações temporais, foram observados coeficientes de correlação significativos, mas sempre com inclusão do mês de março. Estes resultados, especialmente para as regiões da Fronteira Oeste (Região 1), Campanha (Região 2) e Planície Interna (Região 4), estão de acordo com o zoneamento agrícola do arroz irrigado, proposto por Steinmetz et al. (2001).

A Zona Sul (Região 6) não apresentou correlação significativa entre a temperatura mínima e o rendimento, nos períodos analisados, apesar de o mapeamento das probabilidades de ocorrência de temperaturas mínimas do ar, durante o período reprodutivo do arroz irrigado (Steinmetz et al., 2003), indicar que esta região está entre as que apresentam maior probabilidade de ocorrência destas temperaturas.

Por meio da análise das correlações, apresentadas na Tabela 2, nota-se que a radiação solar global e o rendimento de grãos de arroz irrigado apresentam associação nas regiões da Campanha (Região 2), Planície Interna à Lagoa dos Patos (Região 4) e Zona Sul (Região 6). Os coeficientes de correlação apresentados nestas regiões, durante o período reprodutivo, que segundo o calendário médio da cultura para o Rio Grande do Sul vai de janeiro a março, foram $0,365,0,380$ e 0,556 , respectivamente. Os valores das correlações são indicativos de que a radiação solar global pode ser limitante ao rendimento nessas regiões. As demais regiões, com exceção da Depressão Central (Região 3), apresentam pelo menos um período com correlação significativa. Para o Estado como um todo, as correlações foram significativas em praticamente todo o período; esta variável assume importância para a definição do rendimento em todo o ciclo da cultura. As correlações positivas são indicação de que, nos anos com maiores disponibilidades de radiação solar, são obtidos os maiores rendimentos. Para regiões tropicais, Yoshida \& Parao (1976) verificaram que o decréscimo nos rendimentos pode chegar a $70 \%$, caso as plantas de arroz sejam submetidas a baixos níveis de radiação solar. Cabe ressaltar as correlações positivas significativas, observadas nos meses de outubro e novembro, o que pode ser explicado pelo fato de a radiação solar apresentar 

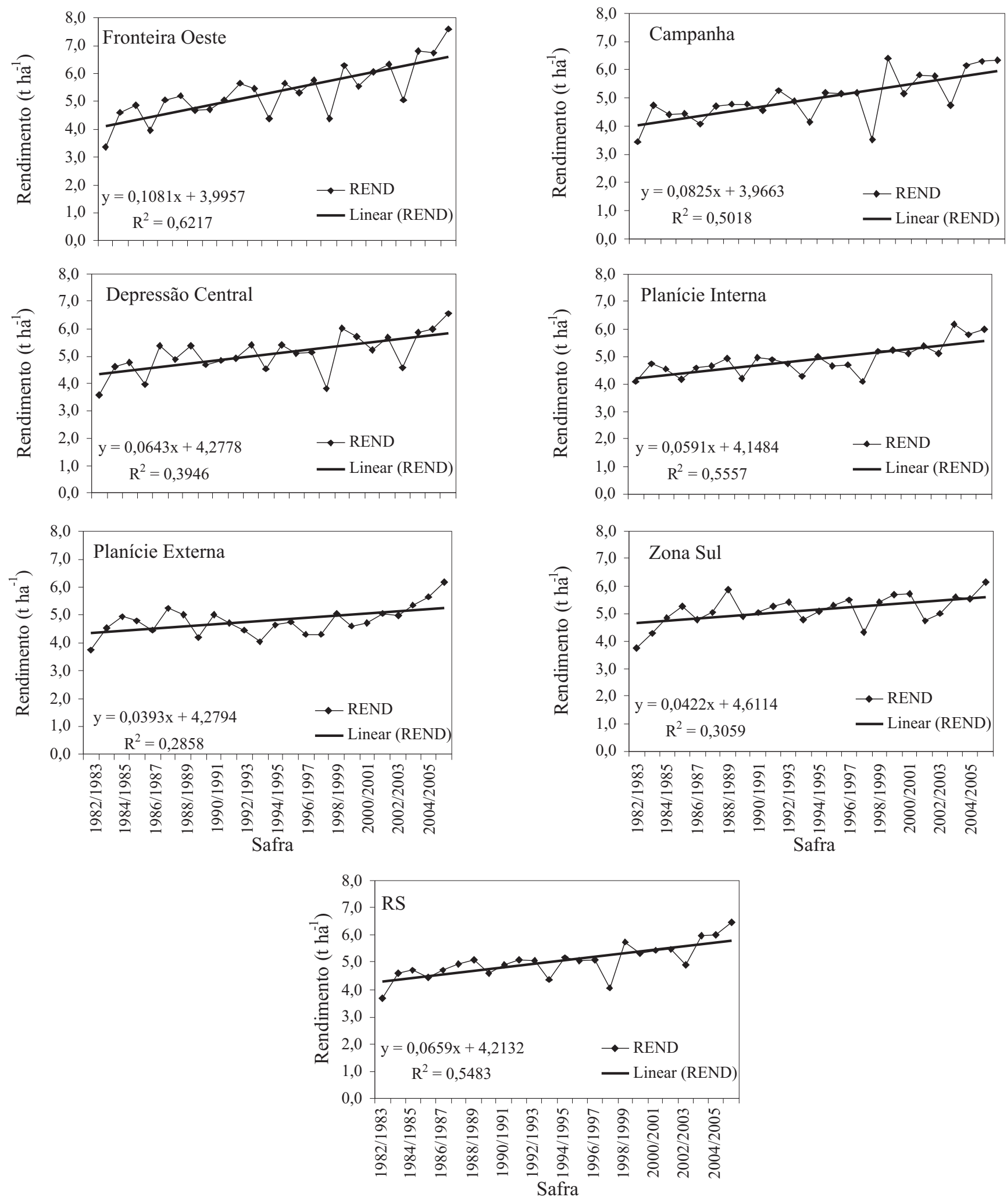

Figura 2. Tendência tecnológica do rendimento de arroz irrigado, no período 1982-2006, para as regiões orizícolas e para o Estado do Rio Grande do Sul (RS). 
correlação negativa com a precipitação pluvial, o que indica que bons níveis de radiação solar, nesse período, possibilitam a semeadura da cultura dentro da época recomendada, além de favorecer o adequado estabelecimento da cultura (Carmona et al., 2002).

Na região da Depressão Central (Região 3), as correlações não significativas (tanto com a radiação solar, quanto com a temperatura mínima), podem estar relacionadas com o fato de as variáveis meteorológicas dessa região serem provenientes apenas de uma estação meteorológica (Santa Maria), e de os rendimentos usados serem os médios de toda a região.
Quanto aos modelos ajustados, verifica-se que as variáveis meteorológicas incluídas foram diferentes em cada região (Tabela 3 ).

Em todos os casos, exceto no modelo ajustado para a Planície Externa à Lagoa dos Patos, a variável radiação solar global foi incluída de alguma forma. Em alguns modelos, a variável selecionada foi apenas a radiação de um mês isolado, enquanto em outros foi selecionada a média dos valores de radiação, obtidos em um determinado período que variou de 2 a 5 meses. Com exceção dos modelos ajustados para as regiões da Depressão Central e Zona Sul, os

Tabela 1. Coeficiente de correlação, entre o número de dias com temperatura igual ou inferior a $15^{\circ} \mathrm{C}$ e o rendimento (corrigido) de grãos, da cultura do arroz irrigado nas diferentes regiões orizícolas do Rio Grande do Sul, no período 1982-2006

\begin{tabular}{|c|c|c|c|c|c|c|c|}
\hline \multirow[t]{2}{*}{ Mês } & \multicolumn{7}{|c|}{ Regiões orizícolas $^{(1)}$} \\
\hline & 1 & 2 & 3 & 4 & 5 & 6 & $\mathrm{RS}$ \\
\hline Dezembro & $0,194^{\mathrm{ns}}$ & $-0,052^{\mathrm{ns}}$ & $0,226^{\mathrm{ns}}$ & $0,133^{\text {ns }}$ & $0,171^{\mathrm{ns}}$ & $-0,188^{\mathrm{ns}}$ & $0,150^{\mathrm{ns}}$ \\
\hline Janeiro & $-0,038^{\mathrm{ns}}$ & $-0,202^{\mathrm{ns}}$ & $0,101^{\mathrm{ns}}$ & $-0,204^{\mathrm{ns}}$ & $0,175^{\mathrm{ns}}$ & $-0,169^{\mathrm{ns}}$ & $-0,183^{\mathrm{ns}}$ \\
\hline Fevereiro & $0,069^{\mathrm{ns}}$ & $-0,002^{\mathrm{ns}}$ & $0,211^{\mathrm{ns}}$ & $-0,033^{\mathrm{ns}}$ & $0,174^{\mathrm{ns}}$ & $0,052^{\mathrm{ns}}$ & $0,060^{\mathrm{ns}}$ \\
\hline Março & $-0,565 * * *$ & $-0,562 * * *$ & $-0,172^{\text {ns }}$ & $-0,533 * * *$ & $-0,465 * *$ & $-0,145^{\mathrm{ns}}$ & $-0,579 * * *$ \\
\hline Janeiro & $0,166^{\mathrm{ns}}$ & $-0,131^{\mathrm{ns}}$ & $0,236^{\mathrm{ns}}$ & $0,011^{\mathrm{ns}}$ & $0,222^{\text {ns }}$ & $-0,218^{\mathrm{ns}}$ & $0,129^{\mathrm{ns}}$ \\
\hline Janeiro-fevereiro & $0,031^{\mathrm{ns}}$ & $-0,110^{\mathrm{ns}}$ & $0,193^{\mathrm{ns}}$ & $-0,133^{\mathrm{ns}}$ & $0,196^{\mathrm{ns}}$ & $-0,077^{\mathrm{ns}}$ & $-0,062^{\mathrm{ns}}$ \\
\hline Fevereiro-março & $-0,461 * *$ & $-0,431 * *$ & $-0,038^{\text {ns }}$ & $-0,466^{* *}$ & $-0,243^{\mathrm{ns}}$ & $-0,098^{\mathrm{ns}}$ & $-0,470 * *$ \\
\hline Dez.-jan.-fev. & $0,165^{\mathrm{ns}}$ & $-0,103^{\mathrm{ns}}$ & $0,278^{\mathrm{ns}}$ & $-0,003^{\mathrm{ns}}$ & $0,248^{\mathrm{ns}}$ & $-0,182^{\mathrm{ns}}$ & $0,121^{\mathrm{ns}}$ \\
\hline Jan.-fev.-mar. & $-0,427 * *$ & $-0,425 * *$ & $0,002^{\mathrm{ns}}$ & $-0,433 * *$ & $-0,129^{\mathrm{ns}}$ & $-0,153^{\mathrm{ns}}$ & $-0,462 * *$ \\
\hline
\end{tabular}

(1)1, Fronteira Oeste; 2, Campanha; 3, Depressão Central; 4, Planície Interna à Lagoa dos Patos; 5, Planície Externa à Lagoa dos Patos; 6, Zona

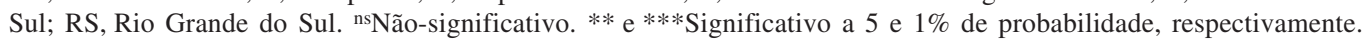

Tabela 2. Coeficiente de correlação entre a radiação solar global e o rendimento de grãos (corrigido), da cultura do arroz irrigado nas diferentes regiões orizícolas do Rio Grande do Sul, no período 1982-2006.

\begin{tabular}{|c|c|c|c|c|c|c|c|}
\hline \multirow[t]{2}{*}{ Mês } & \multicolumn{7}{|c|}{ Regiões orizícolas $^{(1)}$} \\
\hline & 1 & 2 & 3 & 4 & 5 & 6 & $\mathrm{RS}$ \\
\hline Outubro & $0,208^{\mathrm{ns}}$ & $0,372 * *$ & $0,206^{\text {ns }}$ & $0,487 * *$ & $0,189^{\text {ns }}$ & $0,306^{*}$ & $0,410 * *$ \\
\hline Novembro & $0,037^{\mathrm{ns}}$ & $0,532 * * *$ & $0,227^{\mathrm{ns}}$ & $0,382 * *$ & $0,155^{\mathrm{ns}}$ & $0,594 * * *$ & $0,426 * *$ \\
\hline Dezembro & $0,173^{\text {ns }}$ & $0,379 * *$ & $0,285^{\text {ns }}$ & $0,442 * *$ & $-0,070^{\mathrm{ns}}$ & $0,319 *$ & $0,524 * * *$ \\
\hline Janeiro & $-0,156^{\mathrm{ns}}$ & $0,028^{\mathrm{ns}}$ & $-0,042^{\mathrm{ns}}$ & $0,312^{*}$ & $0,051^{\mathrm{ns}}$ & $0,201^{\mathrm{ns}}$ & $0,060^{\mathrm{ns}}$ \\
\hline Fevereiro & $0,315^{*}$ & $0,441 * *$ & $0,114^{\mathrm{ns}}$ & $0,489 * *$ & $0,298^{\mathrm{ns}}$ & $0,487 * *$ & $0,474 * *$ \\
\hline Março & $0,316^{*}$ & $0,226^{\mathrm{ns}}$ & $0,142^{\text {ns }}$ & $0,182^{\mathrm{ns}}$ & $-0,446 * *$ & $0,367^{*}$ & $0,075^{\text {ns }}$ \\
\hline Outubro-novembro & $0,151^{\mathrm{ns}}$ & $0,515 * * *$ & $0,258^{\text {ns }}$ & $0,465 * *$ & $0,206^{\mathrm{ns}}$ & $0,536 * * *$ & $0,512 * * *$ \\
\hline Novembro-dezembro & $0,114^{\mathrm{ns}}$ & $0,548 * * *$ & $0,286^{\mathrm{ns}}$ & $0,431 * *$ & $0,072^{\mathrm{ns}}$ & $0,537 * * *$ & $0,543 * * *$ \\
\hline Dezembro-janeiro & $-0,018^{\mathrm{ns}}$ & $0,219^{\mathrm{ns}}$ & $0,113^{\mathrm{ns}}$ & $0,412 * *$ & $-0,002^{\mathrm{ns}}$ & $0,351 *$ & $0,326^{*}$ \\
\hline Janeiro-fevereiro & $0,111^{\mathrm{ns}}$ & $0,334 *$ & $0,050^{\mathrm{ns}}$ & $0,432 * *$ & $0,225^{\mathrm{ns}}$ & $0,483 * *$ & $0,361 *$ \\
\hline Fevereiro-março & $0,408 * *$ & $0,434 * *$ & $0,149^{\text {ns }}$ & $0,390 * *$ & $-0,172^{\mathrm{ns}}$ & $0,543 * * *$ & $0,398 * *$ \\
\hline Out.-nov.-dez. & $0,181^{\mathrm{ns}}$ & $0,557 * * *$ & $0,293^{\text {ns }}$ & $0,477 * *$ & $0,134^{\mathrm{ns}}$ & $0,510 * * *$ & $0,569 * * *$ \\
\hline Nov._dez.-jan. & $0,006^{\mathrm{ns}}$ & $0,408 * *$ & $0,182^{\text {ns }}$ & $0,416^{* *}$ & $0,084^{\mathrm{ns}}$ & $0,554 * * *$ & $0,436 * *$ \\
\hline Dez.-jan.-fev. & $0,161^{\mathrm{ns}}$ & $0,397 * *$ & $0,135^{\text {ns }}$ & $0,472 * *$ & $0,191^{\mathrm{ns}}$ & $0,534 * * *$ & $0,497 * *$ \\
\hline Jan.-fev.-mar. & $0,238^{\mathrm{ns}}$ & $0,365^{*}$ & $0,088^{\text {ns }}$ & $0,380 * *$ & $-0,127^{\mathrm{ns}}$ & $0,556 * * *$ & $0,329 *$ \\
\hline Out.-nov.-dez.-jan. & $0,073^{\text {ns }}$ & $0,495 * *$ & $0,219^{\mathrm{ns}}$ & $0,455^{* *}$ & $0,138^{\text {ns }}$ & $0,550 * * *$ & $0,503 * *$ \\
\hline Nov.-dez.-jan.-fev. & $0,134^{\mathrm{ns}}$ & $0,495 * *$ & $0,180^{\mathrm{ns}}$ & $0,461 * *$ & $0,212^{\mathrm{ns}}$ & $0,621 * * *$ & $0,534 * * *$ \\
\hline Dez.-jan.-fev.-mar. & $0,266^{\mathrm{ns}}$ & $0,418 * *$ & $0,154^{\mathrm{ns}}$ & $0,421 * *$ & $-0,148^{\mathrm{ns}}$ & $0,582 * * *$ & $0,456 * *$ \\
\hline
\end{tabular}

(1)1, Fronteira Oeste; 2, Campanha; 3, Depressão Central; 4, Planície Interna à Lagoa dos Patos; 5, Planície Externa à Lagoa dos Patos; 6, Zona Sul; RS, Rio Grande do Sul. ns Não-significativo. *** e***Significativo a 10,5 e $1 \%$ de probabilidade, respectivamente. 
modelos também selecionaram alguma expressão do número de dias, em que a temperatura mínima foi igual ou inferior a $15^{\circ} \mathrm{C}$. O mês de março foi considerado em todos os modelos, o que é coerente, pois, pelo calendário agrícola médio da cultura do arroz, é nesse mês que a maioria das lavouras encontra-se no final do período de floração. Isto era esperado, porque março apresentou as maiores correlações com o rendimento em todas as regiões, como mostrado anteriormente.

Os gráficos de dispersão entre os dados oficiais e os estimados, para as diferentes regiões orizícolas e para o Estado, nos períodos de ajuste dos mesmos dados, são apresentados na Figura 3. Os melhores modelos são aqueles que apresentam os menores desvios e as melhores estimativas de valores extremos, isto é, não apresentam tendência de sub ou super estimativa, tanto de altos como de baixos valores de rendimento. Exemplos disso podem ser verificados nos modelos para as regiões de Fronteira Oeste, Campanha e Planície Costeira Interna à Lagoa dos Patos, com os maiores coeficientes de determinação $\left(\mathrm{R}^{2}\right)$ (Tabela 3 ).

No período de teste dos modelos (Figura 4), o coeficiente de correlação entre os dados oficiais e o estimados foi significativo a $5 \%$ de probabilidade apenas para a região da Campanha. As demais regiões não apresentaram coeficientes de correlação estatisticamente significativos, o que, em parte, é decorrência do reduzido número de observações consideradas. A região da Campanha, apesar de ter apresentado um coeficiente de correlação de 0,90 , no ajuste do modelo, e de apresentar o maior coeficiente de correlação entre valores oficiais e estimados $(0,99)$, apresentou tendência de super estimativa dos rendimentos, nos quatro anos utilizados para teste do modelo.
Cabe salientar que os modelos ajustados, em razão de seu caráter empírico, são rigorosamente específicos para as condições das lavouras de arroz irrigado no Rio Grande do Sul. Para outras regiões de cultivo, análises devem ser realizadas para buscar o estabelecimento das relações clima-planta específicas. Para o Estado de Santa Catarina, que apresenta condições climáticas e de manejo das lavouras semelhantes às do Rio Grande do Sul, é provável que se possa usar das mesmas variáveis meteorológicas (radiação solar e número de dias com temperatura inferior a $15^{\circ} \mathrm{C}$ ), como indicadores do rendimento de grãos da cultura. A realização de análise de sensibilidade dos parâmetros dos modelos poderia indicar sua viabilidade de uso em outros estados. Porém, considera-se mais adequado o ajuste de modelos específicos para cada estado, ao invés de se utilizarem modelos gerados com dados de um estado para estimar o rendimento de grãos de arroz em outro. Apesar da limitação de uso dos modelos propostos neste trabalho, restrito ao Estado do Rio Grande do Sul, fica comprovada a possibilidade real de uso desta metodologia para a estimativa de rendimento de grãos dentro de um programa nacional, mas com funções localmente ajustadas.

Uma das alternativas para melhorar o desempenho dos modelos agrometeorológicos é a inclusão de uma componente espectral oriunda de sensores orbitais, pois esta contempla outros fatores, não incluídos no modelo apresentado neste trabalho, que influenciam a definição dos rendimentos como, por exemplo, a ocorrência de pragas, doenças, manejo diferenciado da cultura, fertilidade dos solos, entre outros. No Rio Grande do Sul, para a cultura da soja, pode-se citar os trabalhos de Melo et al. (2003) e Rizzi (2005) com este enfoque.

Tabela 3. Modelos agrometeorológicos para as regiões orizícolas e para o Estado do Rio Grande do Sul, no período $1982-2006$.

\begin{tabular}{llr}
\hline Região & \multicolumn{1}{c}{ Modelo $^{(1)}$} & $\mathrm{R}^{2}$ \\
\hline Fronteira Oeste & $\hat{\mathrm{Y}}=0,108 \mathrm{i}+[4,590-0,102$ (RAjan) $+0,134$ (RAmar) - 0,151 (DImar) $]$ & 0,58 \\
Campanha & $\hat{\mathrm{Y}}=0,082 \mathrm{i}+[1,024+0,183$ (RAnov) $-0,082$ (DImar) & 0,58 \\
Depressão Central & $\hat{\mathrm{Y}}=0,064 \mathrm{i}+[-0,533+0,274$ (RAout-a-dez)] & 0,39 \\
Planície Interna à Lagoa dos Patos & $\hat{\mathrm{Y}}=0,059 \mathrm{i}+[3,141+0,094$ (RAfev) - 0,105 (DIjan-a-mar) $]$ & 0,46 \\
Planície Externa à Lagoa dos Patos & $\hat{\mathrm{Y}}=0,039 \mathrm{i}+[4,546-0,154$ (DImar) & 0,24 \\
Zona Sul & $\hat{\mathrm{Y}}=0,042 \mathrm{i}+[-3,808+0,561$ (RAnov-a-mar) $]$ & 0,41 \\
RS & $\hat{\mathrm{Y}}=0,066 \mathrm{i}+[2,996+0,079$ (RAfev) $-0,038$ (DImar) $]$ & 0,24 \\
\hline
\end{tabular}

(1) $\hat{Y}$ é o rendimento de arroz irrigado $\left(\mathrm{t}_{\text {ha }}{ }^{-1}\right.$ ); i é o ano (o ano 1 corresponde à safra agrícola de 1982/1983); as duas primeiras letras das variáveis independentes, RA e DI, correspondem à radiação solar global e ao número de dias com temperatura mínima inferior a $15^{\circ} \mathrm{C}$, respectivamente; as letras seguintes correspondem ao período da análise (meses). 

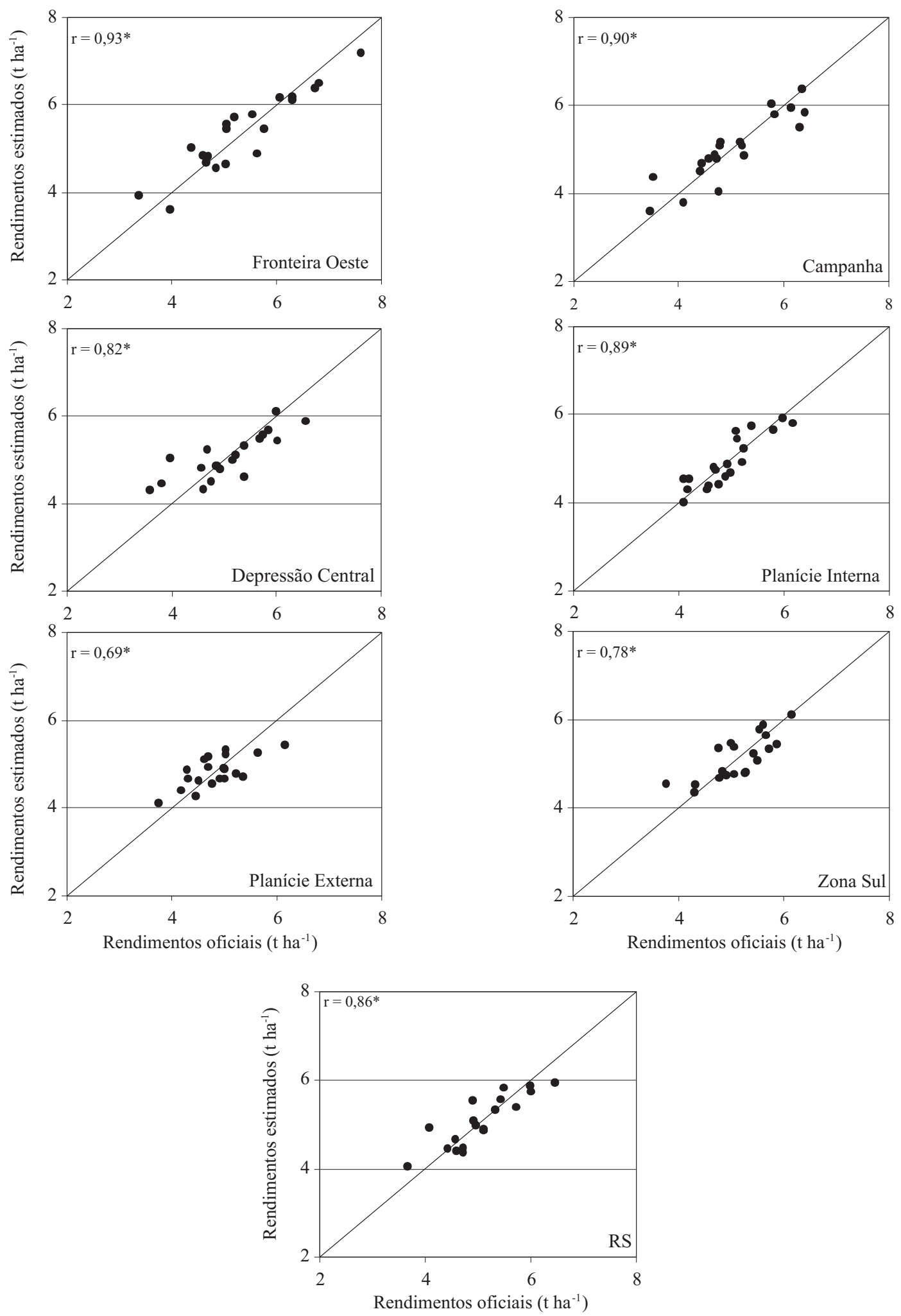

Figura 3. Rendimentos oficiais e estimados pelos modelos agrometeorológicos ajustados, para cada região orizícola do Estado do Rio Grande do Sul, no período de ajuste do modelo. *Significativo a $10 \%$ de probabilidade. 

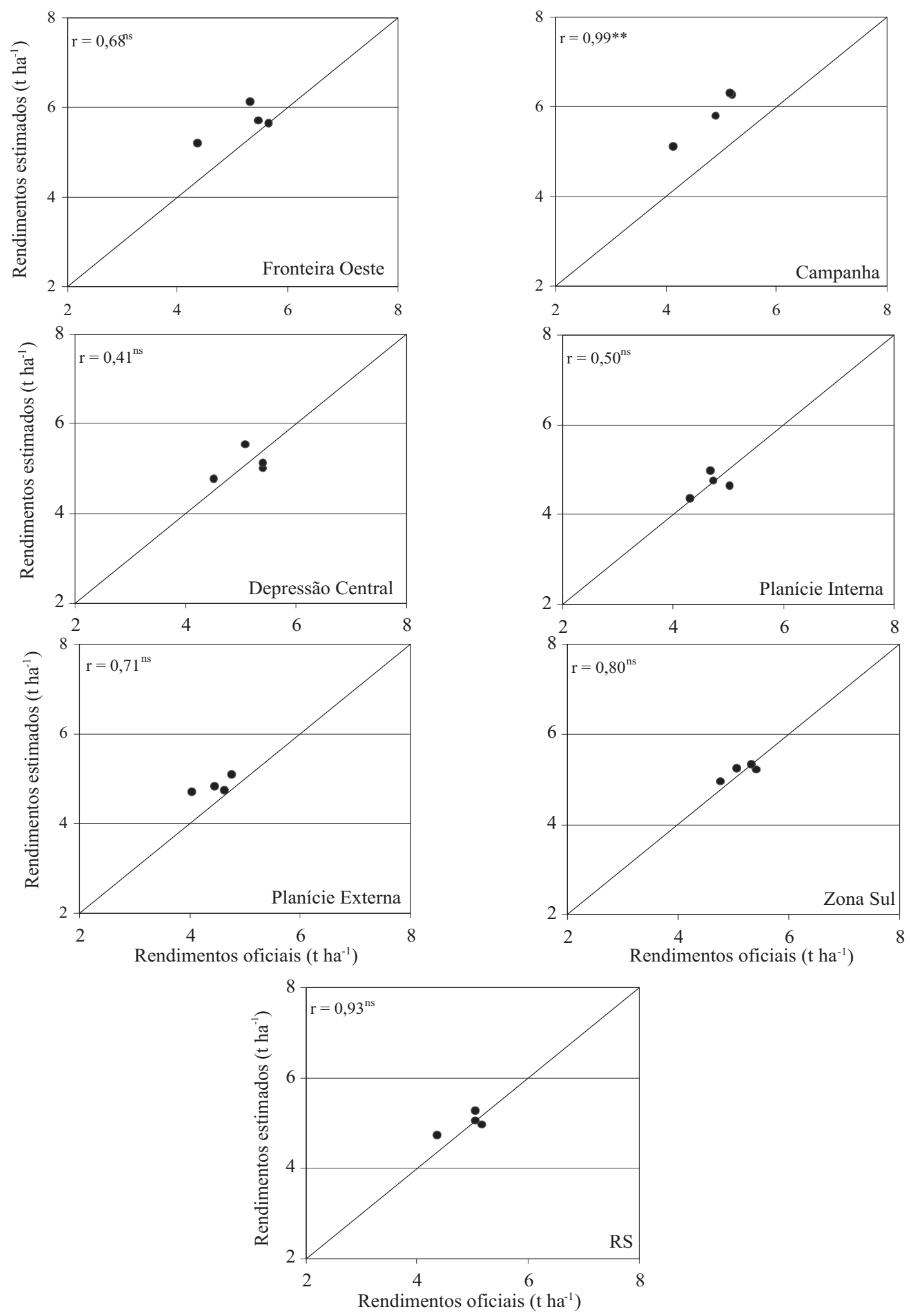

Figura 4. Rendimentos oficiais e estimados pelos modelos agrometeorológicos ajustados, para cada

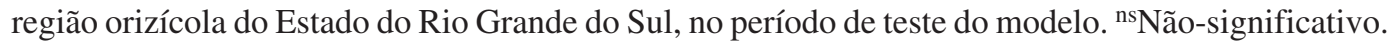
**Significativo a $5 \%$ de probabilidade. 


\section{Conclusões}

1. Existe tendência tecnológica de incremento nos rendimentos de arroz irrigado, tanto nas seis regiões orizícolas, como no Estado do Rio Grande do Sul, o que torna necessária a retirada desta tendência em estudos de modelagem agrometeorológica de rendimento.

2. O número de dias com temperatura mínima do ar menor ou igual a $15^{\circ} \mathrm{C}$ e a radiação solar global são indicadores agrometeorológicos do rendimento de arroz irrigado no Estado do Rio Grande do Sul e respondem por parte da variabilidade dos rendimentos desta cultura.

3. Os modelos agrometeorológicos, de estimativa de rendimento de arroz irrigado no Rio Grande do Sul, apresentam características de precisão, fácil implementação e baixo custo e podem ser introduzidos no programa nacional de previsão de safras.

\section{Referências}

AZAMBUJA, I.H.V.; VERNETTI JÚNIOR, F.J.; MAGALHÃES JÚNIOR, A.M. Aspectos socioeconômicos da produção do arroz. In: GOMES, A.S.; MAGALHÃES JÚNIOR, A.M. (Ed.). Arroz irrigado no Sul do Brasil. Pelotas: Embrapa Clima Temperado; Brasília: Embrapa Informação Tecnológica, 2004. p.23-44.

CARMONA, L.C.; BERLATO, M.A.; BERGONCI, J.I. Relação entre elementos meteorológicos e rendimento do arroz irrigado no Estado do Rio Grande do Sul. Revista Brasileira de Agrometeorologia, v.10, p.289-294, 2002.

FONTANA, D.C.; OLIVEIRA, D. Relação entre radiação solar global e insolação para o Estado do Rio Grande do Sul. Revista Brasileira de Agrometeorologia, v.4, p.87-91, 1996.

HOFFMANN, R.; VIEIRA, S. Análise de regressão: uma introdução à econometria. 3.ed. São Paulo: Hucitec/Edusp, 1998. $379 \mathrm{p}$.

INSTITUTO BRASILEIRO DE GEOGRAFIA E ESTATÍSTICA. Levantamento sistemático da produção agrícola: 2004. Disponível em: www.ibge.gov.br/home/estatistica/indicadores/ agropecuaria/lspa/. Acesso em: 7 nov. 2005.
MATZENAUER, R.; BERGAMASCHI, H.; BERLATO, M.A.; RIBOLDI, J. Modelos agrometeorológicos para estimativa do rendimento de milho, em função da disponibilidade hídrica no Estado do Rio Grande do Sul. Pesquisa Agropecuária Gaúcha, v.1, p.225-241, 1995.

MELO, R.W.; FONTANA, D.C.; BERLATO, M.A. Modelo agrometeorológico-espectral de estimativa de rendimento da soja para o Estado do Rio Grande do Sul. In: SIMPÓSIO BRASILEIRO DE SENSORIAMENTO REMOTO, 11., 2003, Belo Horizonte. Anais. São José dos Campos: Inpe, 2003. p.173-179.

MOTA, F.S. da. Influência da radiação solar e do "frio" no período reprodutivo sobre o rendimento do arroz irrigado em Pelotas e Capão do Leão. Lavoura Arrozeira, v.47, p.22-23, 1994.

NETER, J.; KUTNER, M.H.; NACHTSHEIM, C.J.; WASSERMAN, W. Applied linear regression models. $3^{\text {rd }}$ ed. New York: McGraw Hill, 1997. 720p.

OLDEMAN, L.R.; SESHU, D.V.; CADY, F.B. Response of rice to weather variables. In: INTERNATIONAL WORKSHOP ON THE IMPACT OF WEATHER PARAMETERS ON GROWTH AND YIELD OF RICE, 1986, Los Baños. Weather and rice: proceedings... Los Baños: IRRI, 1986. p.5-39.

RIZZI, R. Geotecnologias em um sistema de estimativa da produção de soja: estudo de caso no Rio Grande do Sul. 2005. 214p. Tese (Doutorado) - Instituto Nacional de Pesquisas Espaciais, São José dos Campos.

STEINMETZ, S. Influência do clima na cultura do arroz irrigado no Rio Grande do Sul. In: GOMES, A.S.; MAGALHÃES JÚNIOR, A.M. (Ed.). Arroz irrigado no Sul do Brasil. Pelotas: Embrapa Clima Temperado; Brasília: Embrapa Informação Tecnológica, 2004. p.45-74.

STEINMETZ, S.; ASSIS, F.N.; BURIOL, G.A.; ESTEFANEL, V.; AMARAL, A.G.; FERREIRA, J.S.A. Mapeamento das probabilidades de ocorrência de temperaturas mínimas do ar, durante o período reprodutivo do arroz irrigado, na metade no Estado do Rio Grande do Sul. Revista Brasileira de Agrometeorologia, v.11, p.169-179, 2003.

STEINMETZ, S.; BRAGA, H.J. Zoneamento de arroz irrigado por épocas de semeadura nos estados do Rio Grande do Sul e Santa Catarina. Revista Brasileira de Agrometeorologia, v.9, p.429-438, 2001.

YOSHIDA, S.; PARAO, F.T. Climatic influence on yield and yield components of lowland rice in the tropics. In: INTERNATIONAL RICE RESEARCH INSTITUTE. Climate and rice. Los Baños: IRRI, 1976. p.471-494.

$\overline{\text { Recebido em } 27 \text { de novembro de } 2007 \text { e aprovado em } 9 \text { de maio de } 2008}$ 